\title{
COVID-19 Social Vaccine Toolkit(C19-SVT)
}

\author{
CRS Kumar \\ Dept of Computer Science and Engineering \\ Defence Institute of Advanced Technology(DIAT) \\ Pune 411025 India \\ Email: suthikshnkumar@ diat.ac.in
}

\begin{abstract}
:
COVID-19 Social Vaccine Toolkit(C19-SVT) addresses social and behavioral modalities which help beat the corona virus. The users become aware of various measures such as social distancing, use of masks, Sanitisation etc. The C19-SVT brings awareness of various prophylactic tasks to combat the COVID-19 Infection. Unlike a biological vaccine, the C19SVT prevents the spread of COVID-19 infection through knowledge dissemination and training skill sets that are essential. A person who has better understanding of how the corona virus spreads, symptoms of the disease, social distancing measures, washing hands, Biological vaccination and other dos and don'ts will be better prepared to prevent this highly infectious disease. Good understanding of fundamentals of COVID-19 preventive measures is the necessity at this critical moment. The C19-SVT is informative, timely, interactive, state of the art tool for the masses.
\end{abstract}

\section{Introduction}

COVID-19 stands for Corona Virus Disease of 2019[5]. The COVID pandemic started in December 2019 in Wuhan, China. It has been spreading world-wide and resulted in Global infection of more than 13 million people and death of more than half a million persons[1] based on WHO Dashboard as on $17^{\text {th }}$ July 2020. The highly infectious COVID-19 has resulted in many lock downs and serious distress. There are many biological vaccines under development[4]. However, till the biological vaccines are developed, inorder to control and contain the spread of COVID-19 infections, the only solution is Social Vaccine. The Social vaccine is for new normal of social and behavioural modalities. Some of the measures of social vaccines are Social distancing, wearing masks, Sanitisation etc.

The Social Vaccine Toolkit for COVID-19 ( C19-SVT) is a specific toolkit addressing the COVID 19 pandemic. It is collection of best practices and lessons from COVID pandemic. The steps and tasks identified are addressing the measures to contain and control the spread of the COVID 19 infection. The various sources from which the prophylactic tasks are collected are highly reliable scientific bodies. The C19-SVT is a compilation of the effective measures for monitoring, modifying and activating social and behavioural modalities. The C91-SVT is continuously evolving with new learning on the emerging COVID-19 pandemic.

C19-SVT is deployed on Microsoft Sway and Microsoft Forms platform for general public access. C19-SVT is a easy to access, browse and understand. The content is presented in a layman language for easy understanding. The scientific explanations for steps suggested are provided inorder to be convincing. This paper is structured as follows: In the next section, C19-SVT architecture is presented along with its components. In section 3, implementation 
and usage of C19-SVT are discussed. The summary and conclusions are presented in section 4.

\section{C19-SVT : Architecture and components}

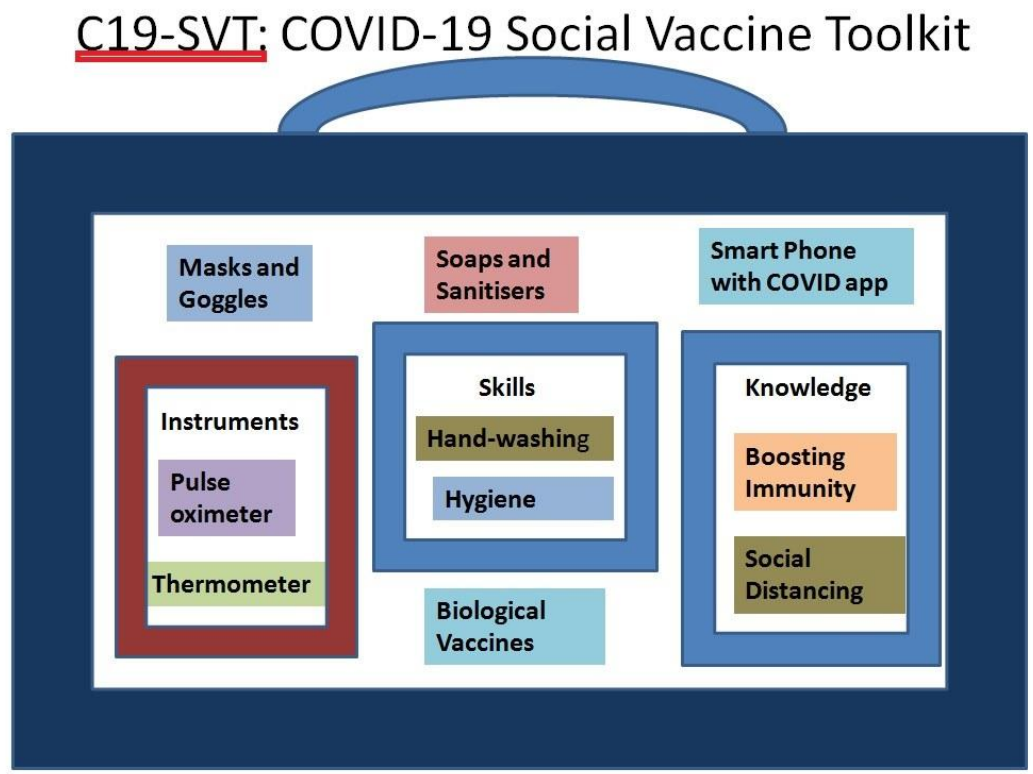

The C19-SVT is proposing multi-pronged strategy which incorporates principles of redundancy and diversity. The C19-SVT is based on scientific approach with solid concepts from reliable sources as the basic components. The C19-SVT robust, fault tolerant architecture is shown in the figure and consists of the following components :

- Face masks: Face masks protect your nose and mouth from getting infected from Corona virus. The corona virus propagates through cough and sneeze droplets from infected patients to others. A person can protect themselves and others by wearing face masks. Corona virus can infect humans through nose, mouth and eyes. Hence it is important to cover mouth and nose with masks and eyes by goggles/spectacles

- Soaps and Sanitisation: Frequent hand washing using liquid soap and water or sanitizer is advised. This is to prevent the virus sticking to hands. The corona virus is killed with soap water or sanitizer and thus when a person touches any surface or object carrying the virus, hand washing will remove the traces of virus. Also, avoid touching nose, mouth and eyes with hands.

- Nasal and Oral Hygiene: Anterior Nasal Washing using Jal-Nethi: This is a technique found to be effective keeping inside of the nose clean through the process of washing using distilled water. The technique is effective when practiced daily. Oral and Throat cleansing using mouth wash and salt water gargling are equally important to keep the virus away.

- Smart Phone with COVID Apps: There are apps released by Governments across the globe to facilitate safety of the users through warnings and exposure notifications. ( Examples : Aarogya Setu[8], ArogyaKshema App[10]). A person who has installed such an app and regularly using is better equipped with the preventive measures. These apps use bluetooth and Cellular networks to keep track of the users and their contacts. Whenever a person is diagnosed with COVID-19, all their contacts are notified about the exposure. 
- Social Distancing: The distancing of $6 \mathrm{ft}$ and above are recommended for all during any social interaction. This is to ensure that the corona virus which is emitted from one person does not reach the other and thus people can stay safe from infections. The action of sneezing and coughing by infected persons can release the droplets with virus in it. These can reach the others nearby and infect them. The social distancing measure thus prevents infection.

- Thermometers: Monitoring the temperature can be useful in containing the spread of infection. The body temperature exceeding 37 degrees celsius indicates fever and the persons are advised to self isolate and get the doctor's advise. The important symptoms of COVID 19 are Fever, Cough and Breathing difficulties.

- Pulse Oximeter: A pulse oximeter measures blood-oxygen saturation. Individuals can keep track of their blood-oxygen saturation levels inorder to monitor any asymptotic COVID 19 infection. The pulse oximeter comes as a handy tool in battling the COVID-19 infection. The oxygen level, when it falls below 95\%, indicates possible infection and consultation with Doctor is recommended.

- Biological Vaccines: Currently, biological vaccines are under development and testing for COVID-19. However, other vaccines such as Hepatitis Vaccines may provide protection as possible adaptive immune cross-reaction. The stimulation of the immune system with currently available vaccines such as Hepatitis vaccine, can be beneficial to prevent further fatalities[11]."Live vaccines have the very broad benefit of going much further than protecting just against the targeted disease" as per Medical Journal publications.

\section{C19-SVT Implementation and Usage}

The C19 is based on Microsoft Sway and made available on the URL[9]. On entering the URL in any standard browser, the following screen is shown

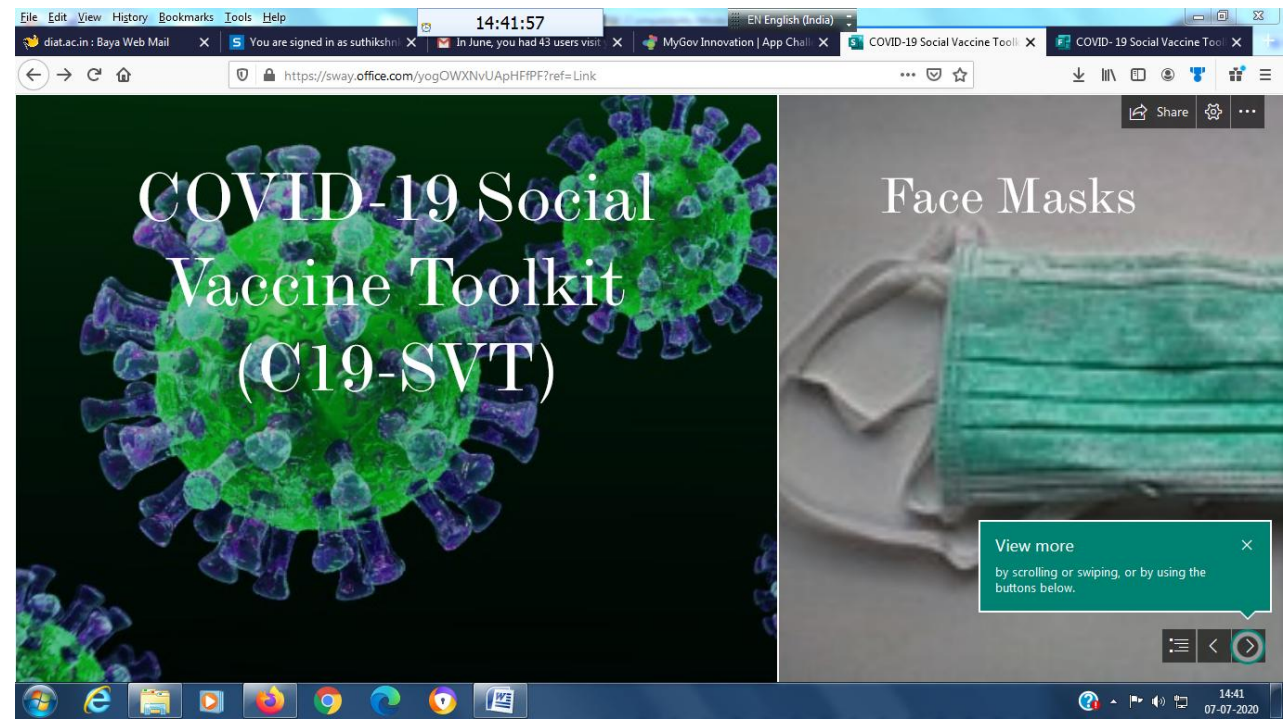

1. Users can navigate from one screen to next using horizontal keys $(<,>)$ or page up/down or arrow key or buttons on bottom right corner.

2. On top right corner, the selection buttons for sharing the link, settings and copying are visible. Users can share/copy etc. 
3. Users once they navigate to last slide, have an option of clicking on a link: "Take a shot of C19-SVT"

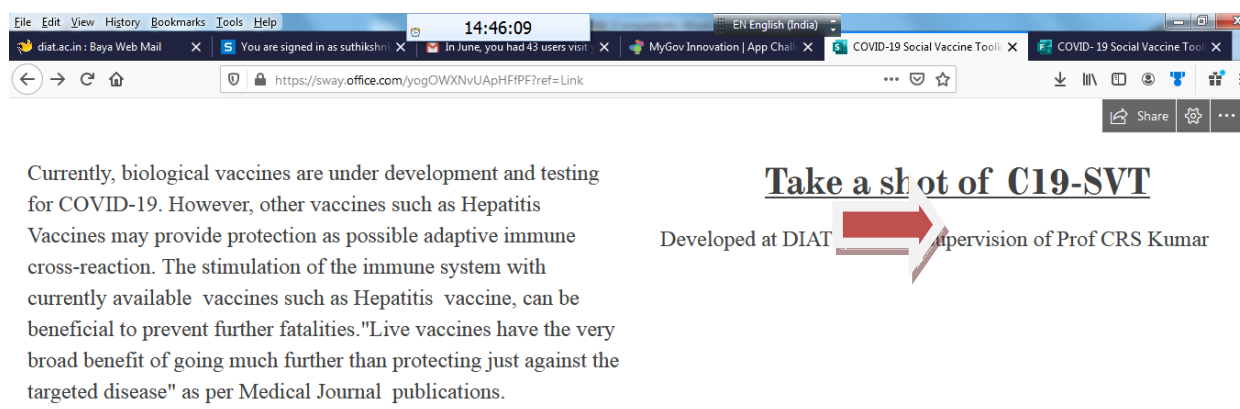

Currently, biological vaccines are under development and testing for COVID-19. However, other vaccines such as Hepatitis

Take a sl ot of C19-SVT

Vaccines may provide protection as possible adaptive immune cross-reaction. The stimulation of the immune system with Developed at DIAT currently available vaccines such as Hepatitis vaccine, can be beneficial to prevent further fatalities."Live vaccines have the very broad benefit of going much further than protecting just against the targeted disease" as per Medical Journal publications.

(4) C

4. The user on clicking the link will be presented with a Microsoft Forms based interactive content :

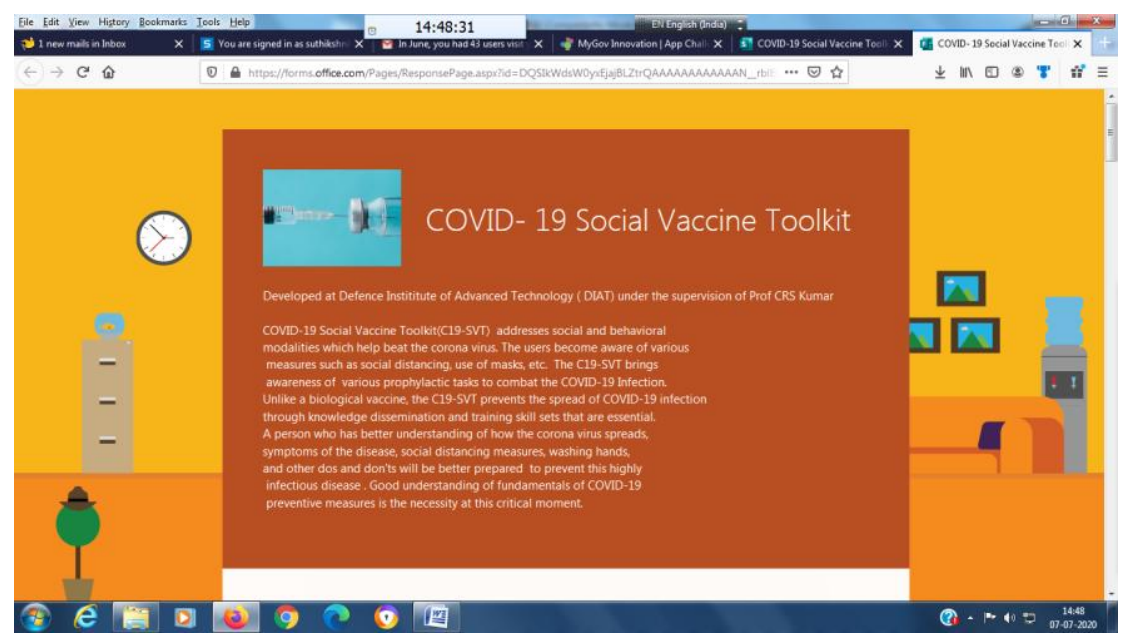

5. The user enters the information they know or their best guesses into the interactive questionnaire. At the end, they need to submit the responses.

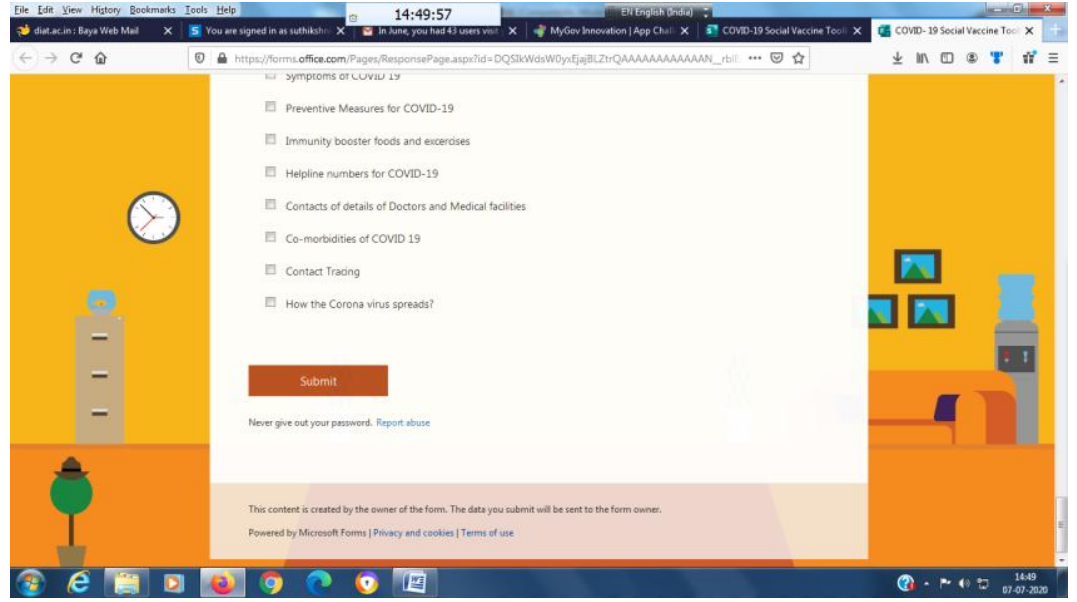


6. The responses from the users will be stored in the database of Mircrosoft Forms and they will be presented with the following screen:

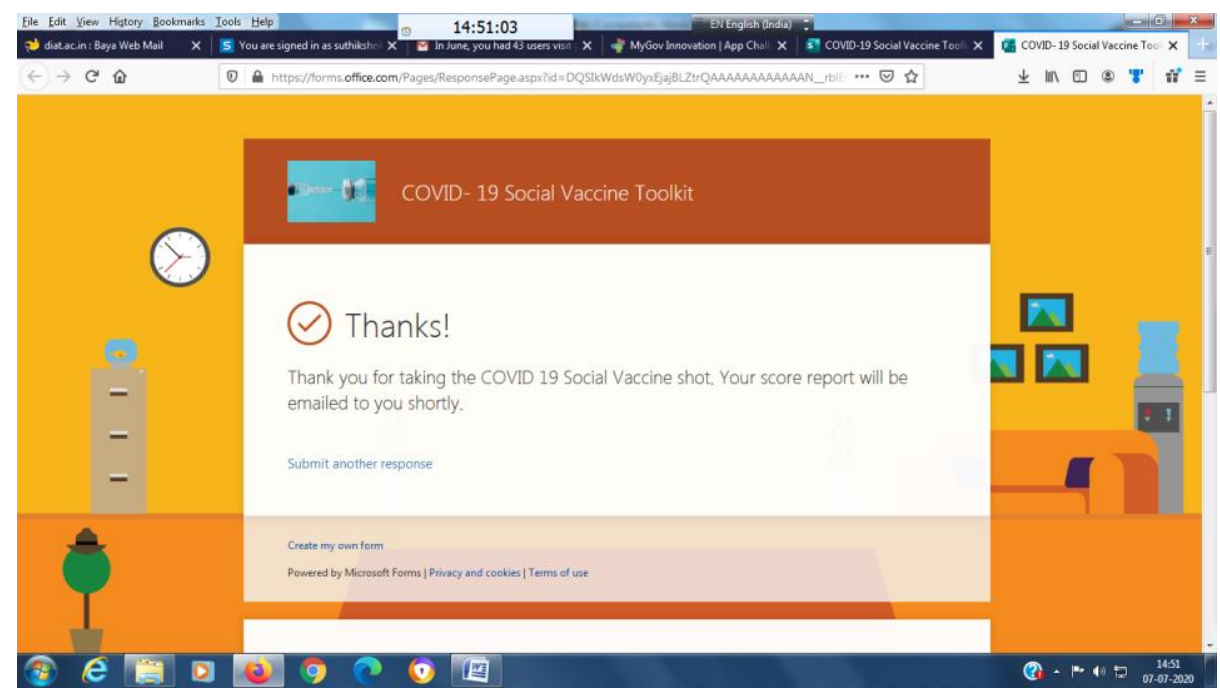

7. The C19-SVT is useful tool for combating the current COVID-19 Pandemic. The C19-SVT has useful and updated information valuable in saving the human life by preventing the spread of Corona virus.

8. The usage of the C19-SVT is monitored through google analytics. The number of users, the country of access, the timings and mode are recorded and displayed as follows. The analytics indicate the growing number of users and increasing popularity of the toolkit.

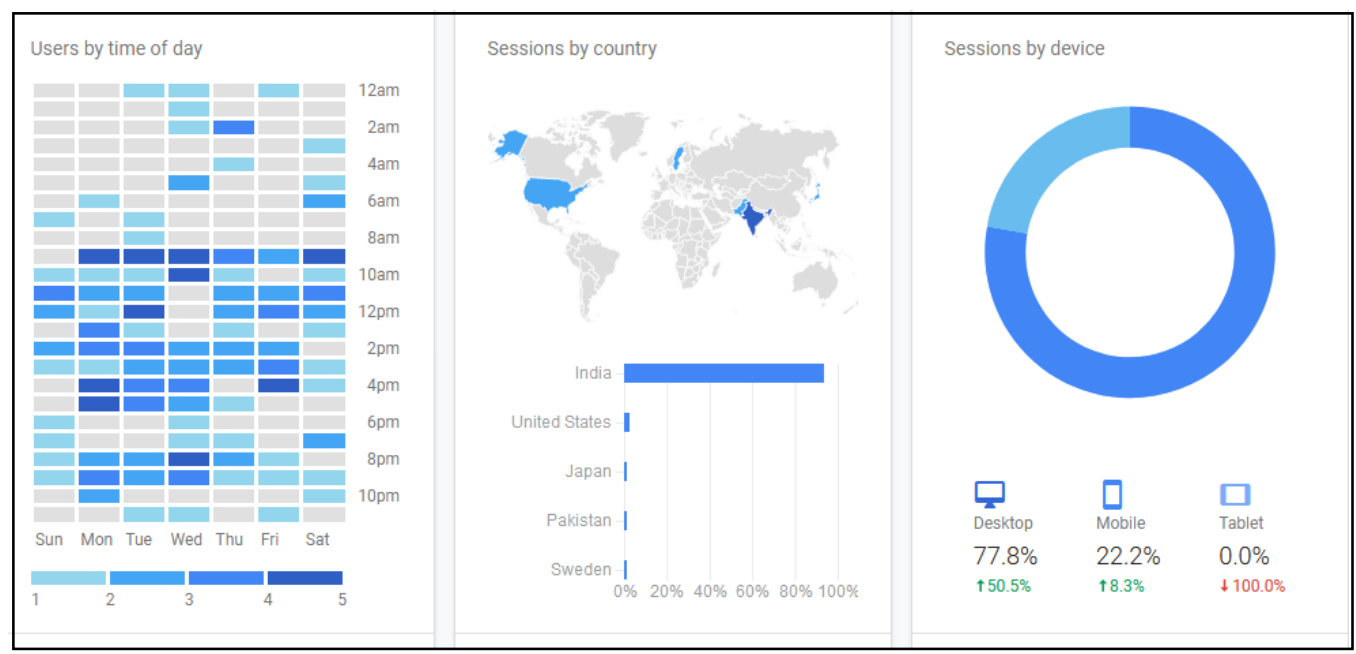

Clinical Trials: The clinical Trials of C19-SVT will be carried out with approvals from Clinical Trial Registry of India(CTRI). This is an observational study. Some of the characteristics of this trial are as follows: 
- CTRI approval for clinical trial requires that Ethics committee need to be formed for monitoring the trials.

- Healthy volunteers from diverse background and age groups are recruited.

- They are given all the information of the C19-SVT and additionally they are given medical advice on immunity boosting and Hepatitis or biological vaccination.

- The volunteers will follow all the instructions such as wearing masks, Social Distancing, Hygiene etc as per the C19-SVT protocols.

- The volunteers report after regular intervals of time their health status.

- The C19-SVT trials are concluded with data analysis and report submission.

C19-SVT Compliance Levels: Depending on the adoption of C19-SVT recommendations for best practices and protocols, 3 levels of compliance can be categorized. The C19-SVT compliance levels are shown in the following table and are useful for data analysis.

\begin{tabular}{|l|l|l|}
\hline $\begin{array}{l}\text { C19-SVT } \\
\text { Compliance } \\
\text { level }\end{array}$ & $\begin{array}{l}\text { Components of C19-SVT Bests } \\
\text { practices, Protocols adopted }\end{array}$ & Remarks \\
\hline 0: Adhoc & None infection \\
\hline 1: Moderate & Masks, Social Distancing, Hand washing & $\begin{array}{l}\text { Moderate Control of Control of } \\
\text { expected }\end{array}$ \\
\hline 2: Good & $\begin{array}{l}\text { Masks, Social Distancing, Hand washing, } \\
\text { Smart Phone with COVID app, } \\
\text { Thermometer in }\end{array}$ & $\begin{array}{l}\text { Good improvement in } \\
\text { Control and Containment of } \\
\text { infection expected. }\end{array}$ \\
\hline 3: Optimized & $\begin{array}{l}\text { Masks and goggles, Social Distancing, } \\
\text { Hand Washing, Nasal and Oral Hygiene, } \\
\text { Smart Phone with COVID app, } \\
\text { Thermometer, Pulse Oximeter, Boosting } \\
\text { Immunity, Biological vaccine }\end{array}$ & $\begin{array}{l}\text { Best Strategy for control of } \\
\text { infection. }\end{array}$ \\
\hline
\end{tabular}

\section{Summary and Conclusions}

C19-SVT is useful, interactive tool for controlling and containing spread of COVID 19 infection. It is educative and knowledge dissemination tool with relevant content. The C19SVT is continuously updated and reviewed by Medical experts. It is accessible for general public free of cost and has global acceptance. The number of users are growing and the usefulness of the C19-SVT is evident from the feedback received. The clinical trials are being initiated to study the effectiveness of C19-SVT. The robust, fault tolerant architecture 
of C19-SVT incorporating best practices and protols with solid scientific basis is the way forward for multi-pronged approach in global fight against COVID-19 infection.

\section{References}

1. WHO COVID-19 Disease Dashboard https://covid19.who.int/ ( Last accessed $16^{\text {th }}$ July 2020)

2. Philip Ball, "Anti-vaccine Movement might undermine Pandemic Efforts", Nature, Vol 581, May 2020.

3. Fran Baum et al., "Social vaccines to resist and change unhealthy social and economic structures: a useful metaphor for health promotion", Health Promotion International, Vol. 24 No. 4, doi:10.1093/heapro/dap026, July 2009.

4. COVID-19 Vaccination, Wikipedia: https://en.wikipedia.org/wiki/COVID19_vaccine ( last accessed 16th July 2020)

5. COVID-19 Pandemic, Wikipedia: https://en.wikipedia.org/wiki/COVID-19_pandemic ( last accessed 16th July 2020)

6. Herd Immunity, Wikipdedia: https://en.wikipedia.org/wiki/Herd_immunity ( last accessed 16th July 2020)

7. Microsoft Sway: https://sway.office.com/ ( Last accessed $16^{\text {th }}$ July 2020)

8. Arogya Setu App: https://www.mygov.in/aarogya-setu-app/ ( Last accessed $16^{\text {th }}$ July 2020)

9. C19-SVT: COVID 19 Social Vaccine Toolkit, https://sway.office.com/yogOWXNvUApHFfPF ( Last accessed 16th July 2020)

10. ArogyaKshema App : COVID 19 Infection Self Assessment Tool: http://14.139.111.180/ArogyaKshema/Arogyakshema.html ( last accessed 16th July 2020)

11. Faik Sarialioglu, Fatma Burcu Belen Apak, Mehmet Haberal, "Can Hepatitis A Vaccine Provide Protection Against COVID-19?", Experimental and Clinical Transplantation (2020) 2: 141-143.

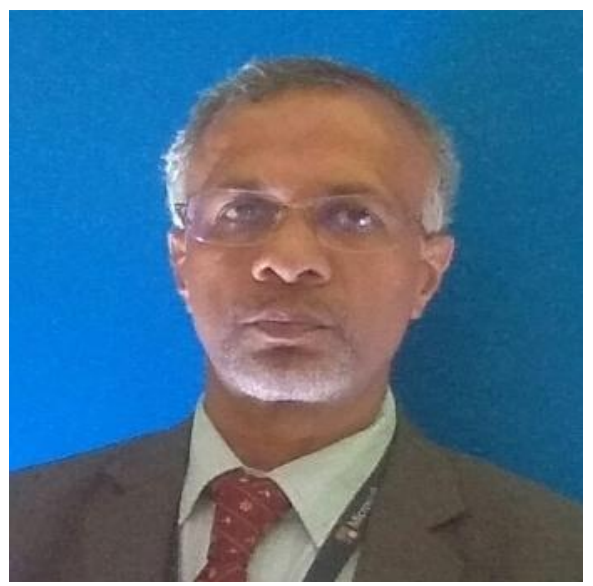

Dr. CRS Kumar is currently Professor in the Computer Science and Engineering Department, Defence Institute of Advanced Technology(DIAT), DRDO, Ministry of Defence. He has received $\mathrm{PhD}$, M.Tech., MBA and B.E. degrees from reputed Universities/Institutes. His areas of interest are in Cyber Security, Network Security, Fault Tolerant Computing, Game Theory, Wireless Networking. He is a Fellow of IETE, Fellow of Institution of Engineers, Senior Member of IEEE, Chartered Engineer(Institution of Engineers) and Distinguished Visitor 
Program(DVP) Speaker of IEEE Computer Society, Lean Six Sigma Green Belt.

Dr Kumar brings with him rich industry, research and academic experience. Dr Kumar has worked in leading MNCs such as Philips, Infineon, L\&T Infotech in senior positions. He has visited several countries such as Australia, Germany, France, Netherlands, USA, UK, HK for work/conference participation. Dr Kumar has won several prizes for innovation and quality. Dr Kumar is member of DIAT Academic council, Board of Management( BoM) and AICTE-INAE Steering Committee. He is currently supervising $7 \mathrm{PhD}$ students and 6 Master's students. He is recipient of several awards including "BestIndividual for Creating Cyber Security Awareness"at CSI-IT2020 Annual Technology Conference 2017, held at IIT Mumbai and "Microsoft Innovative Educator Expert ( MIEExpert) Project Showcase Award" at Microsoft Edu Days 